\title{
Difference of Thrombus Location between Initial Noninvasive Vascular Image and First DSA Findings in Mechanical Thrombectomy for Intracranial Large Vessel Occlusion: Post Hoc Analysis of the SKIP Study
}

\author{
Tetsuhiro HigashidA, ${ }^{1}$ Ryuzaburo KANAZAWA, ${ }^{1}$ Takanori UCHIDA, ${ }^{1}$ \\ Yuichi TAKAHASHI, ${ }^{1}$ Kentaro SUZUKI, ${ }^{2}$ and Kazumi KIMURA ${ }^{2}$ \\ ${ }^{1}$ Department of Neurosurgery, Nagareyama Central Hospital, Nagareyama, Chiba, Japan \\ ${ }^{2}$ Department of Neurology, Nippon Medical School, Tokyo, Japan
}

\begin{abstract}
In patients who undergo mechanical thrombectomy for intracranial large vessel occlusion, the occluded site is sometimes distal to the site shown in the initial vascular imaging. We investigated the factors related to the change in the occluded site between the sequential imagings. The 203 patients in the SKIP study were reviewed retrospectively. Magnetic resonance angiography (MRA) or computed tomography angiography (CTA) was used to assess the occluded site. The occluded site shown in the cerebral angiography appeared to be distal to the occluded site shown in the initial vascular imaging in 55 patients (group A). The location of the occluded site in the remaining 148 patients did not change between the sequential imagings (group B). MRA was used more often than CTA in group A (54 MRA, 1 CTA; $P<0.01$ ). Patients with middle cerebral artery (M1) occlusion were more likely to show change of the occluded site than patients with internal carotid artery (ICA) occlusion (M1: $38 \%$, ICA: $9 \% ; P<0.01)$. The number of patients who received intravenous recombinant tissue plasminogen activator did not differ between the two groups (group A: $54 \%$, group B: $49 \% ; P=0.5$ ). In patients with acute intracranial large vessel occlusion who require mechanical thrombectomy, physicians should be aware that the location of the thrombus may be distal to the occluded site shown in the initial vascular imaging, particularly in patients with M1 occlusion shown by MRA.
\end{abstract}

Keywords: mechanical thrombectomy, MRA, digital subtraction angiography, middle cerebral artery

\section{Introduction}

Mechanical thrombectomy has proved to be effective for patients with acute ischemic stroke caused by large vessel occlusions in the intracranial anterior circulation. ${ }^{1-7)}$ Before mechanical thrombectomy is performed; it is strongly recommended that the architecture and occluded site of the intracranial vessels is evaluated using noninvasive imaging such as computed tomography angiography (CTA) or magnetic resonance angiography (MRA). ${ }^{8)}$ In the subsequent cerebral angiography, the target occluded

Received May 6, 2021; Accepted July 19, 2021

Copyright@ 2021 The Japan Neurosurgical Society This work is licensed under a Creative Commons AttributionNonCommercial-NoDerivatives International License. site for mechanical thrombectomy is sometimes shown to be distal to the occluded site in the initial noninvasive vascular imaging. ${ }^{9)}$ Two hypotheses have been proposed to explain this phenomenon.

First, the occluded site shown in the initial noninvasive vascular imaging may be shown to be proximal to the actual occluded site due to mechanical features of the imaging modality. ${ }^{10-15)}$ Second, the thrombus may have migrated to the distal intracranial vessels before cerebral angiography was performed..$^{10,16-21)}$ It has been recommended that intravenous thrombolysis using recombinant tissue plasminogen activator (rt-PA) is administered within 4.5 hours after the onset of cerebral ischemic stroke, even in cases of mechanical thrombectomy. ${ }^{9)}$ However, rt-PA may facilitate the fragmentation and distal migration of intracranial thrombus, resulting in difficulty in performing the thrombectomy. ${ }^{10,17-20,22-25)}$ 
We recently reported the efficiency and safety of direct mechanical thrombectomy without the administration of rt-PA in patients who were eligible for intravenous rt-PA (SKIP study). ${ }^{26)}$ In the present study, we investigated the patients from the SKIP study who showed the change in the location of the occluded site between the initial noninvasive vascular imaging and the subsequent digital subtraction angiography (DSA), and elucidated the factors related to the change in the occluded site.

\section{Materials and Methods}

The SKIP study was multicenter, prospective, randomized, open-label clinical trial that investigated the efficacy of direct mechanical thrombectomy without administration of intravenous rt-PA. This trial was approved by the institutional review board of each hospital. The study design, methods, and results of the SKIP study have been described previously. ${ }^{26,27)}$ All enrolled patients had acute intracranial large vessel occlusion, specifically in the internal carotid artery (ICA) or M1 segment of the middle cerebral artery (M1). After eligibility to participate in the SKIP study was determined based on clinical data, the occluded site of the intracranial artery was assessed by MRA or CTA. Immediately after ICA or M1 was confirmed to be occluded, the patients were enrolled and randomly assigned to the direct group, which did not receive intravenous rt-PA, or the bridging group, which did receive intravenous rt-PA, and then underwent immediate mechanical thrombectomy. In this procedure, the location of the thrombus causing intracranial large vessel occlusion was assessed using the DSA taken initially. The occluded site was categorized as ICA, proximal M1, or distal M1 and M2 segments of the middle cerebral artery. Proximal M1 was defined as $<5 \mathrm{~mm}$ from the ICA terminus in an anteroposterior view of $3 \mathrm{D}$ reconstructed imaging. The patients in whom the occluded site shown on DSA changed to the distal category from the occluded site shown in the initial noninvasive vascular imaging were categorized as group A. The patients in whom the category of the occluded site did not change between the initial noninvasive vascular imaging and DSA were categorized as group B. A core image judging committee (two expert neurologists, SF and TH) assessed the occluded site independently. ${ }^{28)}$

The favorable outcome in this study was defined as modified Rankin scale $0-2$ at 90 days after onset. Successful recanalization was defined as modified Thrombolysis in Cerebral Infarction score $\geq 2 \mathrm{~b}$ according to angiographic findings after mechanical thrombectomy. ${ }^{29)}$
Table 1 Occluded site and administration of intravenous rt-PA

\begin{tabular}{|c|c|c|c|c|}
\hline & \multirow{2}{*}{$\begin{array}{c}\text { ICA } \\
(n=77)\end{array}$} & \multicolumn{2}{|c|}{ M1 $(n=126)$} & \multirow{2}{*}{$P$ value } \\
\hline & & $\begin{array}{c}\text { Proximal } \\
\text { M1 }(\mathrm{n}=36)\end{array}$ & $\begin{array}{l}\text { Distal M1 } \\
(\mathrm{n}=90)\end{array}$ & \\
\hline $\begin{array}{l}\text { Initial } \\
\text { noninvasive } \\
\text { vascular } \\
\text { imaging }\end{array}$ & & & & 0.2 \\
\hline $\begin{array}{l}\text { CTA } \\
(n=23)\end{array}$ & 12 & 2 & 9 & \\
\hline $\begin{array}{l}\text { MRA } \\
(\mathrm{n}=180)\end{array}$ & 65 & 34 & 81 & \\
\hline IV rt-PA & & & & 0.6 \\
\hline $\begin{array}{l}\text { Yes } \\
(n=103)\end{array}$ & 36 & 18 & 49 & \\
\hline $\begin{array}{l}\text { No } \\
(n=100)\end{array}$ & 41 & 18 & 41 & \\
\hline
\end{tabular}

ICA: internal carotid artery, IV: intravenous, M1: M1 segment of middle cerebral artery, rt-PA: recombinant tissue plasminogen activator.

For statistical analysis, the unpaired t-test was used for continuous variables and the chi-squared test for nominal variables. $P<0.05$ was considered statistically significant.

\section{Results}

From January 2017 through July 2019, 204 patients were enrolled in the SKIP study. One patient was excluded for analysis in this present study because the occlusion was caused by vessel dissection. For the initial assessment of the occluded site, MRA was used in 180 patients $(88.7 \%)$, whereas CTA was used in 23 patients (11.3\%). The occluded site shown in MRA or CTA was ICA in 77 patients (37.9\%) and M1 in 126 patients (62.1\%). After patients were randomly assigned in a $1: 1$ ratio to the group of mechanical thrombectomy with or without intravenous rt-PA, 103 patients received intravenous rt-PA (Table 1). Fifty-five patients were categorized as group A and the remaining 148 patients were categorized as group B. MRA was used more frequently than CTA as the initial noninvasive vascular imaging in group A (54 MRA, 1 CTA; $P<0.01$ ). Patients with M1 occlusion in the noninvasive vascular imaging were categorized more often into group A than patients with ICA occlusion (M1: $38 \%$, ICA: $9 \% ; P<0.01)$. The change in the location of the occluded site between the sequential imagings did not affect the successful recanalization rate and functional outcome (Table 2). 
Table 2 Associated factors and change in occluded sites in sequential imaging

\begin{tabular}{|c|c|c|c|}
\hline & $\begin{array}{l}\text { Distal } \\
\text { change in } \\
\text { occluded } \\
\text { site in } \\
\text { sequential } \\
\text { imaging } \\
\text { (Group A) } \\
\text { (n=55) }\end{array}$ & $\begin{array}{c}\text { No } \\
\text { change in } \\
\text { occluded } \\
\text { site in } \\
\text { sequential } \\
\text { imaging } \\
\text { (Group B) } \\
\text { (n=148) }\end{array}$ & $P$ value \\
\hline $\begin{array}{l}\text { Initial noninvasive } \\
\text { vascular imaging }\end{array}$ & & & $<0.01$ \\
\hline CTA $(\mathrm{n}=23)$ & 1 & 22 & \\
\hline $\operatorname{MRA}(\mathrm{n}=180)$ & 54 & 126 & \\
\hline Occluded site & & & $<0.01$ \\
\hline ICA $(\mathrm{n}=77)$ & 7 & 70 & \\
\hline $\operatorname{MCA}(\mathrm{n}=126)$ & 48 & 78 & \\
\hline $\begin{array}{l}\text { TICI grade } \geq 2 b \\
(\mathrm{n}=187)\end{array}$ & 51 & 136 & 0.9 \\
\hline $\begin{array}{l}\mathrm{mRS} 0-2 \text { at } 90 \text { days } \\
(\mathrm{n}=119)\end{array}$ & 35 & 84 & 0.3 \\
\hline
\end{tabular}

ICA: internal carotid artery, MCA: middle cerebral artery, mRS: modified Rankin scale, TICI: thrombolysis in cerebral infarction.

There were 30 patients in whom DSA showed that the target thrombus for mechanical thrombectomy was located in the M2 segment. It was noted that most of these patients (29/30) showed distal M1 occlusion in the initial noninvasive vascular imaging and that two thirds of patients (20/30) received intravenous rt-PA (Table 3 ).

Although the distal change in the occluded site was seen frequently in patients with distal M1 occlusion who received intravenous rt-PA $(P=0.09)$, the number of patients who received intravenous rt-PA did not differ between the two groups (group A: $54 \%$, group B: $49 \%$; $P=0.5$ ). In patients who received rt-PA, the mean interval from administration of rt-PA to the puncture of cerebral angiography was 8 minutes and did not differ between the two groups. Also, the mean interval from randomization to puncture was 21 minutes and did not differ between the two groups (Table 4).

\section{Discussion}

In this study, the patients with acute M1 occlusion shown in the initial noninvasive vascular imaging, especially by MRA, tended to show a change in the occluded site distally in the subsequent DSA, regardless of rt-PA administration. M1 occlusion and the usage of MRA as an initial assessment tool
Table 3 Detail of occluded site in sequential imaging

\begin{tabular}{llc}
\hline $\begin{array}{l}\text { Occluded site in } \\
\text { initial noninvasive } \\
\text { vascular imaging }\end{array}$ & Occluded site in DSA & $\begin{array}{c}\text { Number } \\
\text { of cases }\end{array}$ \\
\hline ICA (n=77) & ICA (no change) & 70 \\
& Proximal M1 & 3 \\
& Distal M1 & 4 \\
& M2 & 0 \\
Proximal M1 (n=36) & $\begin{array}{l}\text { Proximal M1 (no } \\
\text { change) }\end{array}$ & 17 \\
& Distal M1 & 18 \\
& M2 & 1 \\
Distal M1 (n=90) & Distal M1 (no change) & 61 \\
& M2 & 29 \\
\hline
\end{tabular}

ICA: internal carotid artery, M1: M1 segment of middle cerebral artery, M2: M2 segment of middle cerebral artery.

Table 4 IV rt-PA administration and change in occluded site in sequential imaging

\begin{tabular}{|c|c|c|c|}
\hline & $\begin{array}{l}\text { Distal change } \\
\text { in occluded } \\
\text { site in } \\
\text { sequential } \\
\text { imaging } \\
\text { (Group A) } \\
(\mathrm{n}=55)\end{array}$ & $\begin{array}{c}\text { No change in } \\
\text { occluded site } \\
\text { in sequential } \\
\text { imaging } \\
\text { (Group B) } \\
(\mathrm{N}=148)\end{array}$ & $P$ value \\
\hline IV rt-PA & & & 0.5 \\
\hline Yes $(n=103)$ & 30 & 73 & \\
\hline No $(n=100)$ & 25 & 75 & \\
\hline $\begin{array}{l}\text { Time interval } \\
\text { between IV } \\
\text { rt-PA and DSA }\end{array}$ & $7.7(\mathrm{n}=30)$ & $8.6(n=73)$ & 0.7 \\
\hline $\begin{array}{l}\text { Time interval } \\
\text { between } \\
\text { randomization } \\
\text { and DSA }\end{array}$ & 21.1 & 20.9 & 0.9 \\
\hline
\end{tabular}

IV: intravenous, rt-PA: recombinant tissue plasminogen activator.

of the occluded site were determined to be the major causable factors related to the change in the occluded site. It appeared that the reduced signal intensity of MRA in the vascular segment proximally to the thrombus location was the primary reason for the change in the occluded site.

Previous studies have reported the distal migration of the thrombus before mechanical thrombectomy. ${ }^{10,16-19,21)}$ A specific type of thrombus that contains rich erythrocytes with low components of fibrin is likely to be dissolved by rt-PA. ${ }^{30)}$ Also, it 

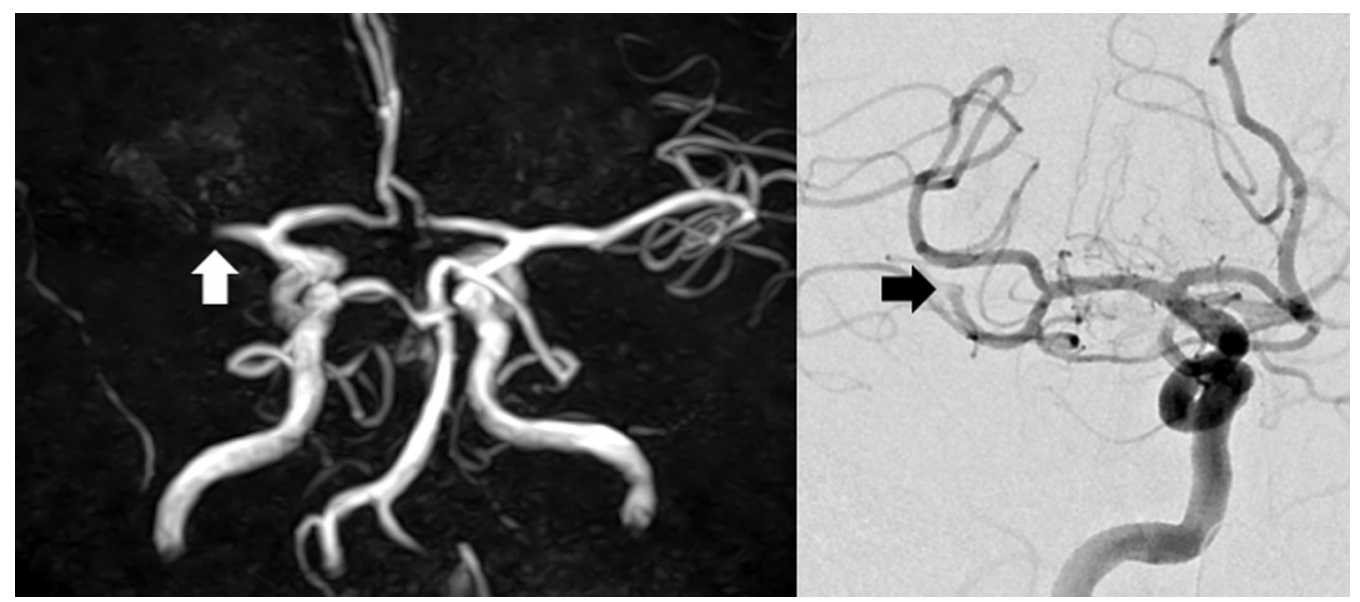

Fig. 1 A representative case showing the discrepancy of the occluded site between noninvasive vascular imaging and DSA. MRA shows the signal loss at M1 segment of the middle cerebral artery (white arrow) (left). The occluded site is shown to be located at the M2 segment in subsequent DSA (black arrow) (right).

is known that thrombi with rich erythrocytes exhibit low friction to the vessel wall and easily migrate distally. ${ }^{10,31,32)}$ It is possible for thrombolysis using intravenous rt-PA to facilitate the fragmentation and distal migration of the thrombus. ${ }^{10,17,18,20,23-25)}$ In some patients, particularly those for whom the time between the initial noninvasive vascular imaging and cerebral angiography was relatively long, the change in the occluded site in DSA may have been caused by distal migration of the thrombus, and the administration of rt-PA may possibly facilitate the migration. Kaesmacher et al. assessed the location of the thrombus by using CTA and subsequent DSA and demonstrated that the frequency of distal migration of the thrombus was not affected by intravenous rt-PA. ${ }^{19)}$ Similarly in the present study, although MRA was mainly selected for the initial assessment of the occluded site, the administration of rt-PA did not increase the number of patients who showed change in the occluded site in DSA. It is notable that the mechanical thrombectomy was usually started immediately after the completion of the initial noninvasive vascular imaging and subsequent administration of rt-PA. Therefore, the reason for the change in the occluded site was not likely to be caused by the distal migration of the thrombus in most of our patients, and other possible factors such as thrombus location, vascular structure, and radiological feature of vascular imagings mainly affect the change in the occluded site. However, distal migration of the thrombus possibly occurred in some patients, particularly with relatively distal occlusion. In this study, there were 30 patients in whom the thrombus was located at the M2 segment shown in DSA. If the thrombus is actually located at the M2 segment, the other patent M2 branch should be clearly seen in the noninvasive vascular imaging, which could accurately identify the occluded site as M2 segment. In addition, most of these cases were initially determined to be distal M1 occlusion in the noninvasive vascular imaging. These findings suggest that the thrombus possibly moved from the distal M1 segment to the M2 segment during the time between the noninvasive vascular imaging and DSA (Fig. 1).

Conversely, the feature of MRA may be a primary reason for the change in the occluded site between the sequential imagings because MRA was used for the initial assessment of the occluded site in most of the patients who showed the change in the occluded site in DSA. Time-of-flight (TOF) MRA is a widely available noninvasive technique that requires no radiation exposure and no administration of contrast agent. When MRI is used as the first-line modality for the assessment of acute ischemic stroke, TOF MRA is recommended to detect the location of the vessel occlusion. However, despite high sensitivity and specificity in identifying the absence of blood flow, TOF MRA tends to overestimate the degree of stenosis and misidentify the occluded sites, typically for distal vascular lesions, due to its lack of sensitivity of slow blood flow and lack of ability of direct visualization of the thrombus. In addition, TOF MRA requires a comparatively long time to complete, leading to patient motion artifacts and degradation of image quality. ${ }^{12,14,15)}$ Contrast-enhanced MRA and black-blood MRI are recently used methods that can improve the ability 
to localize the site of the vessel occlusion because these techniques have low sensitivity to flow dynamics and high spatial resolution. ${ }^{14,33-36)}$ Despite these superior techniques of MRI, CTA has been recommended to evaluate the site of intracranial vessel occlusion in patients with acute stroke due to its higher sensitivity and positive predictive value as well as the shorter acquisition time compared with MRI. ${ }^{14,37,38)}$ Group A has only one patient in whom CTA was selected as the initial assessment of the occluded site. In this patient, CTA showed the occluded site located in the distal M1 segment and the thrombus located in the M2 segment in DSA. Also, this patient received intravenous rt-PA. Considering the high accuracy of CTA for thrombus localization, the change in the occluded site in this patient may have been caused by the distal migration of the thrombus.

In patients with M1 occlusion and a patent anterior cerebral artery, the signal intensity of ICA in TOF MRA should be intact because the blood flow through ICA and the patent anterior cerebral artery is preserved. Therefore, the M1 occlusion is not likely to be incorrectly shown as ICA occlusion even when MRA is used for the assessment of the occluded site. In this study, 188 patients (93\%) had the ipsilateral A1 segment of anterior cerebral artery with antegrade flow seen in DSA. It was noted that the sensitivity of M1 occlusion with MRA was $96 \%$ $(110 / 115)$ in patients with the ipsilateral A1 segment in comparison with the one of $67 \%(4 / 6)$ in patients without the A1 segment $(P=0.03)$. This suggests that MRA is comparatively useful to distinguish between ICA and M1 occlusion in most cases. However, it may be difficult to localize the occluded site precisely at M1 by using TOF MRA, which is highly susceptible to blood flow dynamics, and the signal intensity is likely to be attenuated by slow flow. Indeed, in this study, DSA was more likely to show the different occluded site in patients with M1 occlusion than in those with ICA occlusion. It was noted that DSA detected distal M1 occlusion in half of patients with proximal M1 occlusion shown in the initial noninvasive vascular imaging. In addition, the location of the thrombus in DSA was shown to be at the M2 segment in one-third of patients with distal M1 occlusion shown in the initial noninvasive vascular imaging. Conversely, it was noted that the occluded site rarely changed to the M2 segment in DSA in patients with relatively proximal occlusion (ICA or proximal M1) shown in the initial noninvasive vascular imaging.

In conclusion, in patients with acute intracranial large vessel occlusion who require mechanical thrombectomy, the location of the thrombus may be distal to the occluded site in the preliminarily taken noninvasive imaging, particularly in cases of M1 occlusion shown by MRA. The strategy of mechanical thrombectomy must be modified based on the location of the thrombus. Therefore, physicians should be aware that the target thrombus may be located distally to the occluded site shown in the initial noninvasive vascular imaging.

There were some limitations in this study due to insufficient data. The first limitation was a lack of detailed information about time. In order to discuss the possibility of thrombus migration, it was desirable to use the time interval between the acquisition of initial vascular imaging and DSA rather than the time interval between randomization and puncture of cerebral angiography. However, there was no available exact time of acquiring these radiological pictures in the original database of the SKIP study. Although randomization should be performed immediately after taking the initial noninvasive vascular imaging and it should not take a long time to obtain the first intracranial DSA after the puncture, it was no doubt that the time interval between these radiological pictures was slightly longer than that between randomization and puncture. The second limitation was a lack of detailed information about vascular structure. The anatomical features of M1 segment of the middle cerebral artery such as length, diameter, shape, and location of bifurcated branches might contribute to the change in the occluded site in DSA. Unfortunately, these kinds of information based on the radiological images was also not available in the original database of the SKIP study. Therefore, it was not possible to evaluate if these factors affected the change in the occluded site.

\section{Acknowledgment}

The authors would like to thank all the participants of SKIP study for data acquisition and supporting their work.

\section{Conflicts of Interest Disclosure}

Dr. Suzuki received grants from the Japanese Society for Neuroendovascular Therapy and a scholarship to study abroad from the Uehara Memorial Foundation outside the submitted work. Dr. Kimura received grants from 38th Mihara Cerebrovascular Disorder Research Promotion Fund Ltd, Teijin Pharma Ltd, Medtronic Co Ltd, Pfizer Japan Inc, Daiichi Sankyo Co, and Nippon Boehringer Ingelheim Co Ltd, and personal fees from Daiichi Sankyo Co, Bayer Healthcare Co Ltd, Nippon Boehringer Ingelheim Co Ltd, and Bristol-Myers Squibb Co Ltd 
outside the submitted work. No other disclosures were reported. All authors who are members of The Japan Neurosurgical Society (JNS) have registered online Self-reported COI Disclosure Statement Forms through the website for JNS members.

\section{References}

1) Goyal M, Menon BK, van Zwam WH, et al.: Endovascular thrombectomy after large-vessel ischaemic stroke: a meta-analysis of individual patient data from five randomised trials. Lancet 387: 1723-1731, 2016

2) Saver JL, Goyal M, Bonafe A, et al.: Stent-retriever thrombectomy after intravenous t-PA vs. t-PA alone in stroke. $N$ Engl J Med 372: 2285-2295, 2015

3) Jovin TG, Chamorro A, Cobo E, et al.: Thrombectomy within 8 hours after symptom onset in ischemic stroke. N Engl J Med 372: 2296-2306, 2015

4) Goyal M, Demchuk AM, Menon BK, et al.: Randomized assessment of rapid endovascular treatment of ischemic stroke. N Engl J Med 372: 1019-1030, 2015

5) Campbell BC, Mitchell PJ, Kleinig TJ, et al.: Endovascular therapy for ischemic stroke with perfusion-imaging selection. $N$ Engl J Med 372: 1009-1018, 2015

6) Berkhemer OA, Fransen PS, Beumer D, et al.: A randomized trial of intraarterial treatment for acute ischemic stroke. $N$ Engl J Med 372: 11-20, 2015

7) Hayakawa M, Matsumaru Y, Yamagami H, et al.: Trends in endovascular reperfusion therapy for acute stroke after introduction of mechanical thrombectomy devices: Japanese Registry of NeuroEndovascular Therapy (JR-NET)3. Neurol Med Chir (Tokyo) 60: 191-201, 2020

8) Powers WJ, Derdeyn CP, Biller J, et al.: American Heart Association/American Stroke Association focused update of the 2013 guidelines for the early management of patients with acute ischemic stroke regarding endovascular treatment: a guideline for healthcare professionals from the American Heart Association/American Stroke Association. Stroke 46: 3020-3035, 2015

9) Qureshi AI, Qureshi MH, Siddiq F, Kainth D, Hassan AE, Maud A: Preprocedure change in arterial occlusion in acute ischemic stroke patients undergoing endovascular treatment by computed tomographic angiography. Am J Emerg Med 33: 631-634, 2015

10) Alves HC, Treurniet KM, Jansen IGH, et al.: Thrombus migration paradox in patients with acute ischemic stroke. Stroke 50: 3156-3163, 2019

11) Kappelhof M, Marquering HA, Berkhemer OA, et al.: Accuracy of CT angiography for differentiating pseudo-occlusion from true occlusion or high-grade stenosis of the extracranial ICA in acute ischemic stroke: a retrospective MR CLEAN substudy. AJNR Am J Neuroradiol 39: 892-898, 2018

12) Tong E, Hou Q, Fiebach JB, Wintermark M: The role of imaging in acute ischemic stroke. Neurosurg Focus 36: E3, 2014
13) Marquering HA, Nederkoorn PJ, Beenen LF, et al.: Carotid pseudo-occlusion on CTA in patients with acute ischemic stroke: a concerning observation. Clin Neurol Neurosurg 115: 1591-1594, 2013

14) Bash S, Villablanca JP, Jahan R, et al.: Intracranial vascular stenosis and occlusive disease: evaluation with CT angiography, MR angiography, and digital subtraction angiography. AJNR Am J Neuroradiol 26: 1012-1021, 2005

15) Axel L: Blood flow effects in magnetic resonance imaging. Magn Reson Annu 237-244, 1986

16) Koge J, Matsumoto S, Nakahara I, et al.: Impact of thrombus migration on clinical outcomes in patients with internal carotid artery occlusions and patent middle cerebral artery. J Neurol Sci 412: 116737, 2020

17) Ren Y, Churilov L, Mitchell P, Dowling R, Bush S, Yan B: Clot migration is associated with intravenous thrombolysis in the setting of acute ischemic stroke. Stroke 49: 3060-3062, 2018

18) Kaesmacher J, Kleine JF: Bridging therapy with i. v. rtPA in MCA occlusion prior to endovascular thrombectomy: a double-edged sword? Clin Neuroradiol 28: 81-89, 2018

19) Kaesmacher J, Maegerlein C, Kaesmacher M, et al.: Thrombus migration in the middle cerebral artery: incidence, imaging signs, and impact on success of endovascular thrombectomy. J Am Heart Assoc 6: e005149, 2017

20) López-Cuevas R, Lago A, Tembl JI: Downstream migration and fragmentation of a spontaneous calcific embolus after thrombolysis in a patient with ischemic stroke. J Stroke Cerebrovasc Dis 25: e165e166, 2016

21) Yoon JH, Shin YS, Lim YC, Kim HS, Nam HS, Heo JH: Distal migration of thrombus during intra-arterial thrombolysis. Eur Neurol 63: 62-63, 2010

22) Compagne KCJ, van der Sluijs PM, van den Wijngaard IR, et al.: Endovascular treatment: the role of dominant caliber M2 segment occlusion in ischemic stroke. Stroke 50: 419-427, 2019

23) Mueller L, Pult F, Meisterernst J, et al.: Impact of intravenous thrombolysis on recanalization rates in patients with stroke treated with bridging therapy. Eur J Neurol 24: 1016-1021, 2017

24) Kaesmacher J, Boeckh-Behrens T, Simon S, et al.: Risk of thrombus fragmentation during endovascular stroke treatment. AJNR Am J Neuroradiol 38: 991998, 2017

25) Fischer U, Kaesmacher J, Mendes Pereira V, et al.: Direct mechanical thrombectomy versus combined intravenous and mechanical thrombectomy in large-artery anterior circulation stroke: a topical review. Stroke 48: 2912-2918, 2017

26) Suzuki K, Matsumaru Y, Takeuchi M, et al.: Effect of mechanical thrombectomy without vs with intravenous thrombolysis on functional outcome among patients with acute ischemic stroke: the SKIP randomized clinical trial. JAMA 325: 244-253, 2021

27) Suzuki K, Kimura K, Takeuchi M, et al.: The randomized study of endovascular therapy with versus 
without intravenous tissue plasminogen activator in acute stroke with ICA and M1 occlusion (SKIP study). Int J Stroke 14: 752-755, 2019

28) Hirano T, Sasaki M, Mori E, et al.: Residual vessel length on magnetic resonance angiography identifies poor responders to alteplase in acute middle cerebral artery occlusion patients: exploratory analysis of the Japan Alteplase Clinical Trial II. Stroke 41: 2828-2833, 2010

29) Zaidat OO, Yoo AJ, Khatri P, et al.: Recommendations on angiographic revascularization grading standards for acute ischemic stroke: a consensus statement. Stroke 44: 2650-2663, 2013

30) Choi MH, Park GH, Lee JS, et al.: Erythrocyte fraction within retrieved thrombi contributes to thrombolytic response in acute ischemic stroke. Stroke 49: 652-659, 2018

31) Gunning GM, McArdle K, Mirza M, Duffy S, Gilvarry M, Brouwer PA: Clot friction variation with fibrin content; implications for resistance to thrombectomy. J Neurointerv Surg 10: 34-38, 2018

32) Maegerlein C, Friedrich B, Berndt M, et al.: Impact of histological thrombus composition on preinterventional thrombus migration in patients with acute occlusions of the middle cerebral artery. Interv Neuroradiol 24: 70-75, 2018

33) Al-Smadi AS, Abdalla RN, Elmokadem AH, et al.: Diagnostic accuracy of high-resolution black-blood MRI in the evaluation of intracranial large-vessel arterial occlusions. AJNR Am J Neuroradiol 40: 954-959, 2019
34) Boujan T, Neuberger U, Pfaff J, et al.: Value of contrast-enhanced MRA versus time-of-flight MRA in acute ischemic stroke MRI. AJNR Am J Neuroradiol 39: 1710-1716, 2018

35) Le Bras A, Raoult H, Ferré JC, Ronzière T, Gauvrit JY: Optimal MRI sequence for identifying occlusion location in acute stroke: which value of time-resolved contrast-enhanced MRA? AJNR Am J Neuroradiol 36: 1081-1088, 2015

36) Pedraza S, Silva Y, Mendez J, et al.: Comparison of preperfusion and postperfusion magnetic resonance angiography in acute stroke. Stroke 35: 2105-2110, 2004

37) Tan IY, Demchuk AM, Hopyan J, et al.: CT angiography clot burden score and collateral score: correlation with clinical and radiologic outcomes in acute middle cerebral artery infarct. AJNR Am J Neuroradiol 30: 525-531, 2009

38) Nguyen-Huynh MN, Wintermark M, English J, et al.: How accurate is CT angiography in evaluating intracranial atherosclerotic disease? Stroke 39: 11841188, 2008

Corresponding author: Tetsuhiro Higashida, MD, PhD Department of Neurosurgery, Nagareyama Central Hospital, 2-132-2 Higashihatsuishi, Nagareyama, Chiba 270-0114, Japan. e-mail: tets018@gmail.com 